\title{
Flow spreading behind a combined dam with a through part of tetrahedrons on foothill rivers
}

\author{
Masharif Bakiyev ${ }^{*}$, Azat Khalimbetov, and Otanazar Matkarimov \\ Tashkent Institute of Irrigation and Agricultural Mechanization Engineers, Tashkent, Uzbekistan
}

\begin{abstract}
The areas of the foothill rivers are distinguished by large bottom slopes reaching 0.004 , with increased kinetics of the flow of more than 0.15 , and by gravel-pebble sediments of the river bed and flow. An analysis of the studies performed on the regulatory structures showed that the bulk of them was performed for the conditions of lowland rivers. In the article, a design of a combined dam with a through part of tetrahedrons is proposed. The experiments were conducted in a flume with a rigid bottom and with a variable slope of. The modeling was performed according to Froude in a self-similar area. Experimental studies revealed the presence of two flow spreading modes depending on the bottom slope: a "calm" mode at $i_{\mathcal{A}}<i_{\mathrm{kp}}$ and a "critical" mode at $i_{\mathcal{\lambda}}>i_{\mathrm{kp}}$ These modes are mainly influenced by the degree of constraint and the Froude number. In the previous articles, a solution to the problem was provided for the case $i_{\text {Д }}<i_{\text {кр }}$, which covers the foothill sections of rivers at a "calm" mode. At a further increase in the degree of flow constraint $n>0.3$ and the Froude number $F_{r}>0.15$, a "critical mode" is observed. Here, a solution to the problem for this case is given. The problem, in this case, differs from the previously considered one by non-uniform distribution of velocities in the weakly disturbed core, a significant reduction in the length of the vortex zone; the vertical compression of the flow continues to the end of the vortex zone. The versatility of the velocity distribution in the zones of weakly disturbed core and intense turbulent mixing is experimentally substantiated. With theoretical studies, using the basic equations of applied mechanics, a method for calculating the velocity field was developed, and the planned dimensions of the vortex zones were established. The comparison with experimental data showed satisfactory agreement
\end{abstract}

\section{Introduction}

Stream-bank erosion brings huge losses worldwide to the economies of countries located in coastal zones. Agricultural lands, settlements and cities are being washed away [1-3]. Therefore, it is not surprising that there are many theoretical and experimental studies devoted to improving structures and aimed to develop the methods of the design

*Corresponding author: bakiev1947@ramber.ru 
justification of regulatory structures [4-10]. The researchers paid main attention to determining the depth of local erosion near blind dams [11-15] and the conditions of lowland rivers.

The foothill sections of the rivers have their own features, which consist of the morphology and the hydraulics of the flows. $[16,17]$. On the foothill rivers of Uzbekistan (Zarafshan, Chirchik, Kashkadarya, Akhangaran and others), the slopes of the channel vary within $i=0.001 \div 0.004$, the kinetics of the flow is $F_{r}=0.15 \div 0.5$. Research for these conditions was conducted for blind and through transverse structures [17-19]. In the laboratory of Hydrotechnical Engineering of the Institute, new structures were developed, and large-scale studies of the operation of combined dams with a through part of piles driven into the river bottom were carried out [20-24]. They are the most capital, and the disadvantage is their high cost.

A combined dam is proposed, consisting of a blind part built of local soil and a through part built of reinforced concrete tetrahedrons laid in the dam's head.

Theoretical and experimental studies were carried out in a flume of a variable slope, the physical picture of the flow constrained by a combined dam with a through part of tetrahedrons was revealed, and a method for their calculation was developed.

\section{Materials and Methods}

The experimental research methods were described in detail in our previous articles [1,2]. Here we give the main characteristics of the flow and the channel: the dimensions of the flume, the building factor of the through part $P=0.1 \div 0.4, P=W_{3} / W$ (building area of the through part, the total area), the angle of the dam installation $\alpha_{R}=75 \div 90^{\circ}$, the bottom slope $i_{\text {म }}=0.0001$ before 0.004

Modeling was performed according to Froude. In all experiments, the turbulent mode was maintained. The condition of the planned task $B / h>6$ was met. Water flow rates were measured by the Thompson triangular weir. The free surface was fixed using a measuring needle with leveling. Water velocities were measured with a SANIIRI micro-spinner with a TsISNV-5 electronic sensor. The main provisions of the theory of turbulent jets propagating in a confined space were used in theoretical studies: the scheme of dividing the flow into hydraulic homogeneous zones: a weakly disturbed core, intense turbulent mixing, and reverse currents.

To solve the problem, the basic equations of applied mechanics, law of conservation of momentum in the flow, conservation of the flow rate, and differential equation of nonuniform motion recorded for the transit flow were used, considering the tangential turbulent stresses on the side surfaces, according to Prandtl.

\section{Results and Discussion}

The physical nature of the flow around the combined dam, the through part of which is made of tetrahedrons, has much in common with the flow around the combined dam with the through part from the pile rows [23-28] for flat rivers; though it differs both qualitatively and quantitatively. The flow occurs with the formation of a backwater section between sections F-F and 0-0, planned compression between sections $0-0$ and $\Pi C$, vertical compression between sections ПC-BC, a recovery area between sections BC and Б-Б (Fig. $1)$. 
In the design scheme: beam 0 '- 1 is the boundary between the core and the zone of intense turbulent mixing; 0 ' -2 is the outer boundary of the zone of intense turbulent mixing; 0 '-3 is the boundary of the zone of zero velocities; 0'-4 is the transit flow boundary; МП, ПС; O-O, K-K; В C, В-B - are the sections of the maximum backwater, constraint, planned compression, the end of the vortex zone, vertical compression, common flow state.

The boundaries of zones of intense turbulent mixing 0'-1 C1 $=0.0,0$ '-2 $\mathrm{C} 2=0,37$ were experimentally established (Figure 1)

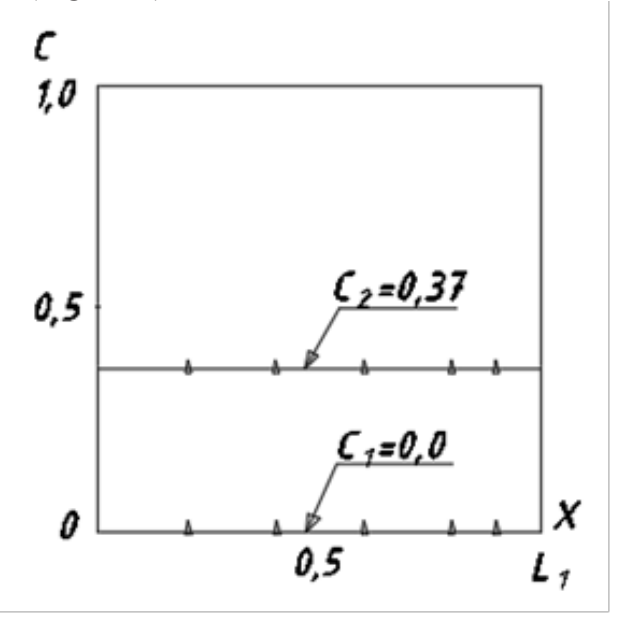

Fig.1. Jet expansion coefficients

It was also found experimentally that the distribution of velocities in the zone of a weakly disturbed core obeys the theoretical Schlichting - Abramovich dependence (Fig. 2).

$$
\frac{u-u_{*}}{u_{\max }-u_{*}}=\left(1-\eta^{1.5}\right)^{2}, \text { where } \eta=\frac{y_{1}-y}{B_{\Omega}}
$$

and the distribution of velocities in the zone of intense turbulent mixing (Fig. 3) is

$$
\frac{u_{\max }-u}{u_{\max }-u_{\mu}}=\left(1-\eta^{1.5}\right)^{2} ; \quad \text { where } \quad \eta=\frac{y_{2}-y}{b}=\frac{y_{2}-y}{y_{2}-y_{1}}
$$




$$
\frac{u-u_{*}}{u_{\max }-u_{*}}
$$

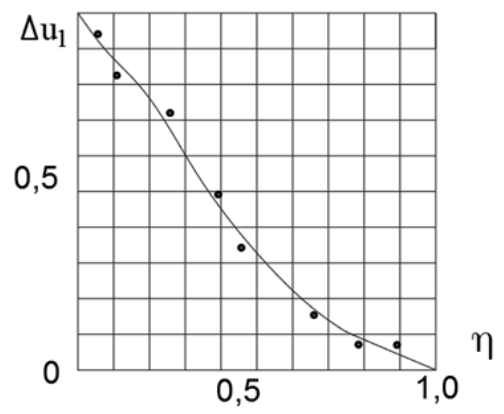

Fig.2. Dimensionless velocity profiles in the core

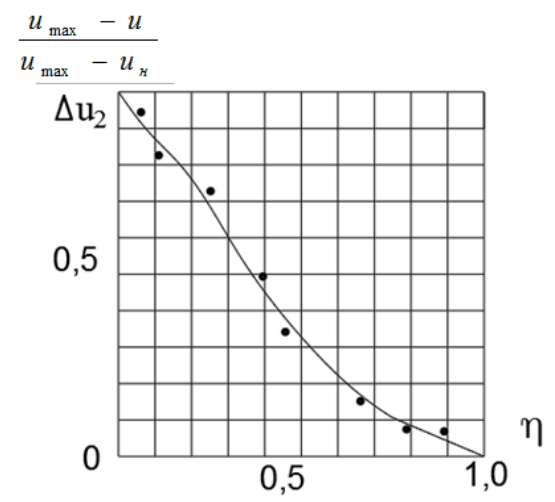

Fig.3. Dimensionless velocity profiles in the region of intensive turbulent mixing

Theoretically, it was necessary to evaluate the influence of the longitudinal slope and other characteristics of the flow and structure on the patterns of change in the maximum velocities in the core ${ }^{u_{\text {aax }}}$, along the opposite bank ${ }^{u_{*}}$ and the determination of the vortex zone length $\mathrm{L}_{\mathrm{B}}$. 


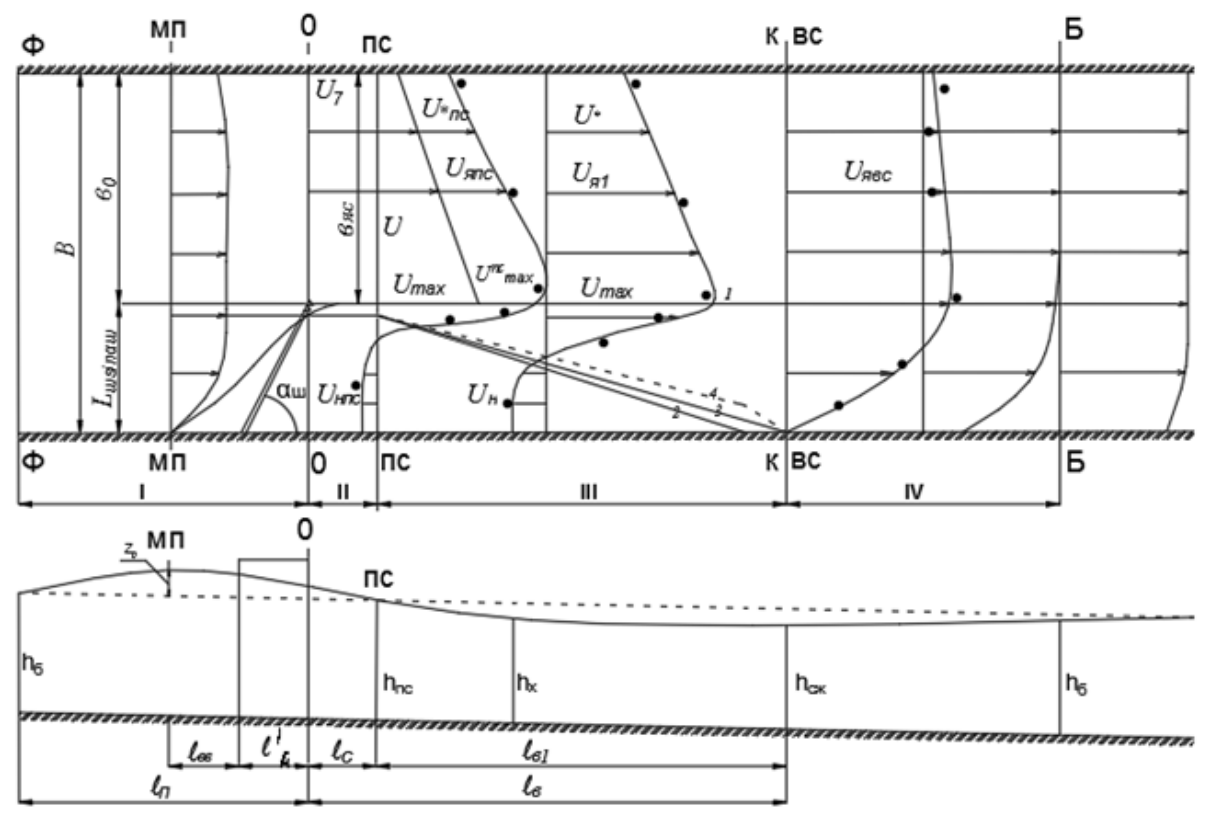

Fig. 4. Diagram of the flow deformed by a transverse dam on the foothill sections of rivers (a "critical" mode)

To determine $u_{\text {Max }}$, we use the equation of conservation of momentum in the flow, written for the cross-sections of $\Pi \mathrm{C}$ and $\mathrm{X}-\mathrm{X}$

$$
\begin{aligned}
& \rho h_{n c} \int_{O}^{\beta_{я c}} u^{2} d y+\rho h_{n c} \int_{y_{1}}^{y_{2}} u^{2} d y+\rho u_{\mathrm{Hпc}}^{2} h_{\text {пс }}\left(B-\beta_{\text {яnс }}-\beta_{c}\right)=\rho h_{x} \int_{O}^{\beta_{я c}} u^{2} d y+\rho h_{x} \\
& \int_{y_{1}}^{y_{2}} u^{2} d y+\rho u_{\mu x}^{2} h_{x}\left(B-\beta_{g x}-6\right)+\rho \int_{0}^{x} \int_{0}^{\beta_{g x}+\beta} \frac{\lambda u^{2}}{2} d y \cdot d x+\frac{\gamma B}{2}\left(h_{x}^{2}-h_{n c}^{2}\right)-\frac{\gamma}{2}\left(h_{n c}+h_{x}\right) B i_{\text {Д }} x
\end{aligned}
$$

where the depth of the flow in the $\mathrm{X}-\mathrm{X}$ section is determined as

$$
h_{x}=h_{n c}+i_{\text {Д }} x-i x=h_{n c}+x\left(i_{g}-i\right)=h_{n c}+I x ; \quad I=i_{\text {Д }}-i
$$

where I is the average value of the depth increment in the spreading section

$$
\frac{\partial h}{d x}=I=\frac{h_{n c}-h}{\ell_{B}}
$$

Performing the integration considering the given dependencies, we obtain

$$
\begin{aligned}
& u_{\max . n c}^{2} \theta_{s c} h_{n c}\left(0.316+0.268 m_{n c}+0.416 m_{n c}^{2}+0.416 u_{\max . n c}^{2} \theta_{c} h_{n c}\right)= \\
& =h_{x} \beta+\frac{\lambda}{2} \int_{o}^{x} \beta d x+\frac{g B}{2}\left(h_{\mathrm{x}}^{2}-h_{n c}^{2}\right)-\frac{g B}{2}\left(h_{n c}+h_{x}\right) i_{g} x
\end{aligned}
$$


Where

$$
\begin{gathered}
\beta=\int_{c}^{B_{r x}+6} u^{2} d y \quad F_{1}=0.316+0.268 m_{n c}+0.416 m_{n c}^{2} \\
m_{n c}=\frac{u_{* n c}}{u_{\max . n c}}
\end{gathered}
$$

The general solution of the integral equation has the form

$$
\beta=-2 g B I \frac{\left(h_{n c}+I x\right)}{4 I+\lambda}-g B i_{g} \frac{x^{2}}{2\left(h_{n c}+I x\right)}+c\left(h_{n c}+I x\right)^{-\left(1+\frac{\lambda}{2 I}\right)}
$$

The constant $\mathrm{c}$ is determined from the boundary conditions at $\mathrm{x}=0$

$$
\beta=u_{\max . n c}^{2}\left(B_{s c} \mathrm{~F}_{1}+0.416 \boldsymbol{B}_{c}\right)
$$

Then from (5)

$$
\beta=-2 g B I \frac{h_{n c}}{4 I+\lambda}+c h_{n c}^{-\left(1+\frac{\lambda}{2 I}\right)}
$$

Hence,

$$
\begin{gathered}
c=\frac{\beta+2 g B I \frac{h_{n c}}{4 I+\lambda}}{h_{n c}^{-\left(1+\frac{\lambda}{2 I}\right)}}= \\
=\frac{u_{\max . n c}^{2}\left(B_{r c} \mathrm{~F}_{1}+0.416 \theta_{c}\right)+2 g B I \frac{h_{n c}}{4 I+\lambda}}{h_{n c}^{-\left(1+\frac{\lambda}{2 I}\right)}}
\end{gathered}
$$

Substituting it into (5), we obtain a partial solution

$$
\beta=-2 g B I \frac{\left(h_{n c}+I x\right)}{4 I+\lambda}-g B i_{\mu} \frac{x^{2}}{2\left(h_{n c}+I x\right)}+\left[u_{\text {max. } n c}^{2}\left(B_{n c} \mathrm{~F}_{1}+0.4166_{c}\right)+2 g B I \frac{h_{n c}}{4 I+\lambda}\right] \frac{\left(h_{n c}+I x\right)^{-\left(1+\frac{\lambda}{2 I}\right)}}{h_{n c}^{-\left(1+\frac{\lambda}{2 l}\right)}}
$$

On the other hand,

$$
\beta=\int_{c}^{\beta_{я x}+6} u^{2} d y=u_{\max }^{2}\left(B_{r} \mathrm{~F}_{2}+0.416 b\right)
$$

where 


$$
\begin{aligned}
& F_{2}=0.316+0.268 m_{1}+0.416 m_{1}^{2} \\
& m_{1}=\frac{u_{*}}{u_{\max }} \\
& u_{\max }^{2}\left(\theta_{\Omega} \mathrm{F}_{2}+0.4168\right)=u_{\max . n c}^{2}\left(B_{\Omega c} F_{1}+0.416 b_{c}\right) \frac{h_{n \mathrm{c}}^{1+\frac{\lambda}{2 I}}}{\left(h_{n c}+I x\right)^{1+\frac{\lambda}{2 I}}}+ \\
& +2 g B I \frac{h_{n c}}{(4 I+\lambda)} \cdot \frac{h_{n c}^{\left(1+\frac{\lambda}{2 I}\right)}}{\left(h_{n c}+I x\right)^{\left(1+\frac{\lambda}{2 I}\right)}}-2 g B I \frac{\left(h_{n c}+I x\right)}{4 I+\lambda}-g B i_{g} \frac{x^{2}}{2\left(h_{n c}+I x\right)}
\end{aligned}
$$

Where

$$
M=\vec{B}_{я c} \mathrm{~F}_{1}+0.416 \vec{B}_{c}
$$

We check at $\mathrm{X}=0$

$$
\left(\frac{u_{\max }}{u_{\max . n \mathrm{c}}}\right)^{2}=\frac{\overrightarrow{\boldsymbol{B}}_{g c} \mathrm{~F}_{1}+0.416 \overrightarrow{\boldsymbol{B}}_{c}}{\left.\overrightarrow{\boldsymbol{B}}_{я c} \mathrm{~F}_{1}+0.416 \overrightarrow{\boldsymbol{B}}_{c}\right)} \quad u_{\max }=u_{\max . n \mathrm{c}}
$$

Suppose we neglect the last term that considers the value of the component of the fluid weight and assume a uniform distribution of velocities in the weakly disturbed core F1 $=1$. In that case, we arrive at the dependence obtained earlier [7].

Equation of conservation of flow rate for sections PS and X-X is written as ПС и X-X

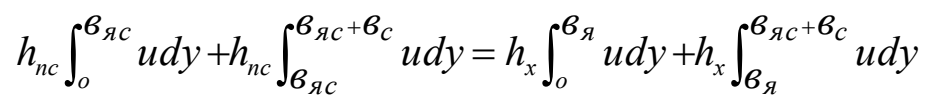

Integrating it with (1) and (2), we obtain

$$
\begin{aligned}
& u_{\max . n c} \theta_{9 c} h_{n c}\left(0.45+0.55 m_{n c}\right)+0.55 u_{\max . n c} \theta_{c} h_{n c}= \\
& =u_{\max } \theta_{\Re} h_{x}\left(0.45+0.55 m_{1}\right)+0.55 u_{\max } 8 h_{x}
\end{aligned}
$$

where

$$
m_{1}=\frac{u_{*}}{u_{\max }}
$$


from (9) we obtain

$$
\frac{u_{\max }}{u_{\max . n c}}=\frac{\left[\vec{\theta}_{g c}\left(0.45+0.55 m_{n c}\right)+0.55 \beta_{c}\right]}{\left[\vec{\theta}_{я}\left(0.45+0.55 m_{1}\right)+0.55 \vec{b}\right](1+\mathrm{I \kappa} \zeta)}
$$

Solving (9) and (6) together, we arrive at a quadratic equation for $m_{1}$

$$
A_{1} m_{1}+A_{2} m_{1}+A_{3}=0
$$

Where

$$
\begin{aligned}
& A_{1}=0.416 \vec{B}_{g} \cdot N^{2}-0.3025 \vec{B}_{g}^{2}(1+\text { ІК } \zeta)^{2} R \\
& A_{2}=0.268 \vec{B}_{g} \cdot N^{2}-(1+I \kappa \zeta)^{2} R \vec{B}_{9}\left(0.495 \vec{B}_{я}+0.605 \vec{B}\right) \\
& A_{3}=\overrightarrow{\boldsymbol{B}}_{\mathrm{g}} \cdot N^{2}(0.316+0.416 \overrightarrow{\boldsymbol{B}})-\left(0.45 \overrightarrow{\boldsymbol{B}}_{\mathrm{я}}+0.55 \overrightarrow{\boldsymbol{B}}\right)^{2}(1+I \kappa \zeta)^{2} R \\
& N=\vec{B}_{\text {яс }}\left(0.45+0.55 m_{n c}\right)+0.55 \vec{B}_{\mathrm{c}} \quad R=\frac{M+P}{(1+I \kappa \zeta)^{1+\frac{\lambda}{2 I}}}-P(1+\mathrm{IK} \zeta)-\frac{i_{Д} \mathrm{~K}^{2} \zeta^{2}}{2 F r_{n c}(1-n)} \\
& \left.M=\vec{b}_{s c} \mathrm{~F}_{1}+0.416 \vec{b}_{\mathrm{c}}\right) ; \quad P=\frac{2 I}{F r_{n c}(4 I+\lambda)(1-n)}, \quad m_{1}=\frac{u_{*}}{u_{\max }}, \quad m_{n c}=\frac{u_{* n c}}{u_{\max . n c}}
\end{aligned}
$$

In equation (10), a root less than one is taken as the main root, and a root greater than one is discarded as it contradicts the physics of the phenomenon.

The length of the vortex zone is determined from the equation of non-uniform motion recorded with account for the tangential turbulent stresses on the side surfaces [1].

The order of the solution remains the same, so we write it down finally as

$$
\begin{aligned}
& L_{\beta}=\frac{A}{E} \ln \frac{\boldsymbol{\theta}_{\mathrm{TBc}}}{\boldsymbol{B}_{\mathrm{Tnc}}} \sqrt{\frac{\mathrm{D} \boldsymbol{\theta}_{\mathrm{Tnc}}+E}{D \boldsymbol{\theta}_{\mathrm{TBc}}^{2}+E}} \\
& A=2 \alpha Q^{2} h_{c \mathrm{p}} ; D=-2 g i_{\mathrm{cp}} h_{c \mathrm{p}} ; E=Q^{2}\left(\frac{\lambda_{\delta} h_{\mathrm{cp}}}{\mathrm{B}_{\mathrm{cp}}}+\lambda_{\text {म }}+2.88 \chi^{2} \frac{h_{\mathrm{cp}}}{B_{\mathrm{cp}}}-4 \alpha I\right) \\
& h_{\mathrm{cp}}=\frac{\mathrm{h}_{n c}+\mathrm{h}_{c}}{2} ; \quad B_{\mathrm{cp}}=\frac{\beta_{1 n c}+\boldsymbol{B}_{18 c}}{2} ; \quad B_{c \mathrm{p}}=\frac{\boldsymbol{B}_{\mathrm{TBc}}+\boldsymbol{B}_{\mathrm{T} n c}}{2} ; \chi=0.21
\end{aligned}
$$

$\lambda_{\sigma} ; \lambda_{\text {Д }}$ are the resistance factors of the bank and bottom

The design of a combined dam is considered, the blind part of which is made of local soil and the through part is made of tetrahedrons. Such a dam combines the positive features of the blind and through structures. Because the through part does not require driving into the bottom of the reservoir, construction costs are significantly reduced. 
The features of such structures operation were experimentally revealed. The formation of two flow modes was established: a "calm" mode at $n_{Д}<0.3, F_{r}<0.15$ and a "critical" mode at $n_{д}>0.3, F_{r}>0.15$. The second flow scheme is considered here. A jet character of the flow around a combined dam with a through part of tetrahedrons was stated. It was found that in the zone of a weakly disturbed core, the distribution of velocities in the plan has a non-uniform character and obeys the theoretical Schlichting - Abramovich dependence (Fig. 2).

The distribution of velocities in the zone of intense turbulent mixing is universal and obeys the theoretical Schlichting-Abramovich dependence (Figure 3).

The boundaries of zones of intense turbulent mixing $0^{\prime}-1 \mathrm{C}_{1}=0.0,0^{\prime}-2 \mathrm{C}_{2}=0.37$ were experimentally established (Figure 1)

A method for calculating the field of flow velocities constrained by a combined dam with a through part of tetrahedrons for the conditions of the foothill sections of rivers was developed. The task is implemented for the second "calm" mode using the integral relation characterizing the law of conservation of momentum, the equations of conservation of flow rate and non-uniform motion recorded, taking into account the tangential turbulent stresses on the side surfaces of the vortex according to Prandtl. Calculated dependencies are obtained to determine the change in the maximum velocities in the weakly disturbed core $U_{*}$ at the opposite bank $U$ and the length of the vortex zones in the spreading area $\ell_{B 1}, \ell_{B 2}$. Direct calculations and their comparison with the experimental data (Fig. 4) showed the correctness of the theoretical solutions obtained.

\section{Conclusions}

1. A change in the longitudinal slope of the bottom, typical for foothill rivers areas, from $i_{D}=0.002$ to $i_{D}=0,004$ leads to a change in the hydraulic and kinematic characteristics of the flow. At $n>0.3$ and the Froude number $F_{r}>0.15$, a "critical" mode is observed.

2. In the presence of a "critical" mode, the distribution of velocities in the zone of a weakly disturbed core is non-uniform and obeys the theoretical Schlichting-Abramovich dependence.

3. The expansion coefficient of the zone of intense turbulent mixing is 0.37 versus 0.27 in the theory of turbulent jets. The distribution of velocities in this zone is also universal.

4. The section of the maximum vertical compression coincides with the end of the vortex zone.

5. Using the main provisions of the theory of turbulent jets, a method for calculating the velocity field and dependence for determining the length of the vortex zone are proposed.

\section{References}

1. A.Khalimbetov, M.Bakiev, S.Shukurova, J.Choriev, X.J.Xayitov Study of submountain river flow patterns constrained by a combined dam https://iopscience.iop.org/article/10.1088/1755-1315/614/1/012053/pdf

2. S.E.Shukurova, A.B.Khalimbetov, M.R.Bakiev, X.J.Xayitov. Deflection of the dynamic axes of flow contracted by combined dam with tetrahedron through-flow part 
3. Istomina, M.N. Complex evaluation of large floods around the world and their negative consequences. K.t.n. dissertation. Moscow, www.dissercat.com/ content/kompleksnaya-otsenka-krupnykh-navodnenii-v-mire-i-ikh-negativnykhposledstviiixzz2KEdJaIBU (2005)

4. Altunin S.T. Channel control. Selhozizdat. p.351, Moscow, (1962)

5. Bazarov D.R. Scientific justification of new numerical methods for design of deformations of river channels, made of easily erodible soils, Tashkent. (2000)

6. Bakiev M.R., Togunova N.P. Hydraulic design of through-flow dikes with varying build-up. Hydraulic construction. 12, pp 14-17, (1989)

7. Bakiev M.R. Improving the structure, design justification methods and design of control structures. Author's abstract to d.t.s. dissertation. Moscow. p.57, (1992)

8. Ikramova M.R. Impact of plane forms of transverse dams on the hydraulic of contracted flow. Author's abstract to k.t.n. dissertation. Tashkent. p.26, (1991)

9. Azinfar Hossein \&Kells A. James, Flow resistance due to a single spur dike in an open channel//Journal Of Hydraulic Research Published Online: p.26, Apr (2010)

10. Jennifer G. Duan, Ph.D., P.E., Mean Flow and Turbulence around a Laboratory Spur Dike//Journal of Hydraulic Engineering October Volume 135, Issue 10 (803 - 811) Online publication date: February 23. (2009)

11. Kayumov O.A. Study of the kinematic structure of the flow and local erosion in massive transverse spurs, Abstract of diss30 p (1969)

12. Lodhi A.S., Jain R.K. \& Sharma P.K., Influence of cohesion on scour around submerged dike founded in clay-sand-gravel mixtures//ISH Journal Of Hydraulic Engineering Published Online: 27 Aug (2015)

13. Mohammad Vaghefi; Masoud Ghodsian; and Seyed Ali Akbar Salehi Neyshabouri, Experimental Study on Scour around a T-Shaped Spur Dike in a Channel Bend// Journal of Hydraulic Engineering/ Volume 138 Issue 5 - May (2012)

14. Roger A. Kuhnle; Carlos V. Alonso; and F. Douglas Shields, Geometry of Scour Holes Associated with $90^{\circ}$ Spur Dikes//Journal of Hydraulic Engineering/Volume 125 Issue 9 - September (1999)

15. Roger A. Kuhnle; Carlos V. Alonso; and F. Douglas Shields, Jr., Local Scour Associated with Angled Spur Dikes//Journal of Hydraulic Engineering Volume 128, Issue 12 (1087 - 1093) Online publication date: November 15.

16. Schulz V.L. Rivers Central Asia. Part2, Leningrad, Gidrometiozat, p.691, (1965)

17. Artamanov K.F. Regulatory structures for water intake on rivers in foothill areas. Frunze, A.I.Kirgiz. 341 p (1963)

18. Bakiev M.R., Khudayberganov X.O. Patterns of flow spreading behind a transverse dam in foothill sections of rivers. J. Architecture, construction, design. 4 Tashkent pp. 48-54.

19. Ishayev F.Sh. Guidelines for the design of rational structures on the rivers of the foothill Central Asia SANIIRI, p.123, Tashkent (1974)

20. Bakiev M.R. River bed regulation by cross combined dikes. XXIV JAHR congress Madrid a study of streams and water sheds of high hydraulic irregularity, 9-13 september, MADRID/ESPANA. (1991)

21. Kadirov O. Improving structures and developing a method for hydraulic calculation of combined dams. Abstract of diss.c.t.s. p.27, (1991)

22. Muradov R. Improving the designs and methods of calculation justification of partially flooded combined dams. Abstract of diss. c.t.n. p.27, Tashkent (1993)

23. Bakiev M.R. Shukurova S.E. Floodplain correction by varingbuild.upcombind dikes. European science review. 7-8, july-August pp. 230-232. (2016) 
24. Bazarov D., Vatin N., Obidov B., and Vokhidov O. Hydrodynamic effects of the flow on the slab of the stand in the presence of cavitation. IOP Conf. Ser. Mater. Sci. Eng. 1030, 012110 (2021).

25. Bazarov D., Markova I., Norkulov B. and Vokhidov O. Hydraulic aspects of the layout of head structures during water intake from lowland rivers. IOP Conf. Ser. Mater. Sci. Eng. 1015, 012041 (2021).

26. Bazarov D., Markova I., Sultanov S. and Kattakulov F. Dynamics of the hydraulic and alluvial regime of the lower reaches of the Amudarya after the commissioning of the Takhiatash and Tuyamuyun hydrosystems. IOP Conf. Ser. Mater. Sci. Eng. 1030, 012110 (2021).

27. Bazarov D. and Vokhidov O. Extinguishing Excess Flow Energy in Spillway Structures. In book: Proceedings of EECE 2020, LNCE 150, pp. 535-545, (2021) DOI: 10.1007/978-3-030-72404-7_52

28. Bazarov D., Markova I., Norkulov B., Isabaev K., Sapaeva M. Operational efficiency of water damless intake. IOP Conf. Ser. Mater. Sci. Eng. 869(7), 072051, (2020)

29. Shukurova C.E., Bakiev M.R. Designing unilateral combined dams. Monograph, p.122, Tashkent, (2019)

30. Bakiev M.R., Kakhharov U.A., Jakhonov A.A., Panjiev S. Flow parameters in the area compressed by transverse floodplain dams. IOP Conference Series: Materials Science and Engineering, 883(1), 012016 (2020)

31. Bakiev, M.R., Kakhharov, U.A., Jakhonov, A.A., Matkarimov, O. Kinematic characteristics of the flow, in the compression region, with bilateral symmetric restriction by floodplain dams. IOP Conference Series: Materials Science and Engineering, 869(7), 072017, (2020)

32. Obidov B., Vokhidov O., Tadjieva D., Kurbanova, U., Isakov A. Hydrodynamic effects on the flow elements of the downstream devices in the presence of cavitation. IOP Conf. Ser. Mater. Sci. Eng. 1030, 012114 (2021).

33. Krutov A., Choriev R., Norkulov B., Mavlyanova D. and Shomurodov A. Mathematical modelling of bottom deformations in the kinematic wave approximation. IOP Conf. Ser. Mater. Sci. Eng. 1030, 012147 (2021).

34. Krutov A., Norkulov B., Uljaev F., and Jamalov F. Results of a numerical study of currents in the vicinity of a damless water intake. IOP Conf. Ser. Mater. Sci. Eng. 1030, 012121 (2021).

35. Krutov A., Norkulov B., Mavlyanova D. Simulation of spreading of non-conservative passive substances in water bodies. IOP Conf. Ser. Mater. Sci. Eng. 883(1), 012028 (2020)

36. Krutov A., Norkulov B., Nurmatov P., Mirzaev M. Applicability of zero-dimensional equations to forecast nonconservative components concentration in water bodies. IOP Conf. Ser. Mater. Sci. Eng. 883(1), 012028 (2020)

37. Krutov A., Norkulov B., Artikbekova F., Nurmatov P. Optimal location of an intake at a reservoir prone to salt diffusion. IOP Conf. Ser. Mater. Sci. Eng. 869(7), 072020, (2020)

38. Shokirov B., Norkulov B., Nishanbaev Kh., Khurazbaev M., Nazarov B. Computer simulation of channel processes. E3S Web of Conferences, 97, 05012, (2019) 